\title{
In Honor of Professor Demetrios A. Spandidos
}

As Editor in Chief of Critical Reviews in Oncogenesis, I have taken the initiative to invite Dr. Apostolos Zaravinos to edit a special issue dedicated to Professor Spandidos' achievements. I am indeed very happy and proud of the publication of this special issue in honor of Dr. Spanididos' long-standing achievements and milestones in the field of cancer. Dr. Spandidos has been a pioneer in scientific investigations and in the recognition and discovery of cancer genes that play pivotal roles in the initiation, differentiation, progression, and maintenance of cancer. These investigations were the result of a long career that began with Dr. Spandidos' postdoctoral fellowship and his discovery of certain cancer genes that are today called "oncogenes." His initial discovery and subsequent discoveries have resulted in leading investigations worldwide in the field of oncogenes and tumor suppressor genes in a variety of human cancers, and these investigations have resulted in a myriad of research publications in highly referenced scientific journals. In addition, the fields of oncogenes and tumor suppressor genes have led to the development of various therapeutic strategies targeting such genes for treatment purposes. Dr. Spandidos' scientific publications exceed 500 (including books and research articles), and of these, more than 300 are listed in PubMed.org.

In addition to his numerous research publications, he has also initiated a series of scientific forums yearly on cancer that have unique attributes in both their scope and their rationales. Such scientific forums contribute significantly to the scientific community at large. Such conferences have been highly successful in promoting scientific collaborations and in supporting the exposure of new scientific milestones to participants worldwide and, in particular, from under-developed countries. In addition to his research publications, Dr. Spandidios has also initiated a series of scientific journals in the field of cancer whose themes are unique and continue to contribute significantly to the scientific community. Dr. Spandidos has also been an excellent mentor for $\mathrm{PhD}$ students, postdoctoral fellows, and medical doctors. Today, the majority of those trainees occupy highly prestigious positions at academic institutions.

I have had the personal pleasure to know Dr. Spandidos for many years, and I have established significant collaborations with him and his laboratory. In addition, he has also recommended several of his fellows to join my laboratory at UCLA for further training. The fellows recommended by Dr. Spandidos were talented, committed, and highly productive in their research and scientific publications.

I hold Dr. Spandidos in very high esteem as a friend, and this special issue in his honor is a token of appreciation and an acknowledgement to his important contributions and impacts in the fields of cancer biology, biochemistry, genetics, and therapeutics. Such contributions are clearly attributable to his research achievements. I am confident that Dr. Spandidos will continue to lead the field and generate many milestones of high significance. I am very proud to be a member of his friendship family and wish him all the best, personally and for his family.

Benjamin Bonavida, $\mathrm{PhD}$

Editor in Chief

Distinguished Research Professor

Department of Microbiology, Immunology, and Molecular Genetics

University of California, Los Angeles 